\title{
SYSTEM DEVELOPMENT OF TRANSMITTING CONFORMAL SATCOM ARRAY ANTENNA STRUCTURES(CSAAS)
}

\section{EMUS 2020}

\author{
M.-S. KIM ${ }^{*}$, J.-W. SEO ${ }^{\dagger}$ \\ Agency for Defense Development (ADD) \\ Yuseong-gu, Daejeonshi, 34186, South Korea \\ e-mail: castle@add.re.kr, http://add.re.kr \\ † e-mail: jwseo@add.re.kr, http://add.re.kr
}

Key words: Tile Antenna, Housing, Radome, CSAAS

\begin{abstract}
This study presents the development results of Conformal SATCOM Array Antenna Structure(CSAAS) for transmitting. Tile Type antenna with $8 \times 8$ antenna element array has been developed instead of reflector antenna. $8 \times 8$ tile antennas are arrayed on the curved surface with one directional curvature to simulate aircraft skin configuration. Housing has the grid structure to minimize out-of-plane deformation that affects the antenna performances. Tile antennas are attached to the surfaces between grids. Radome is designed as an A-sandwich type to carry some distributed skin load and glass epoxy material with lowest dielectric constant and loss tangent was used for the best electromagnetic performance. One tile antenna is designed with a separate data control and power connection for vertical and horizontal polarization. The static structural strength of the housing and impact strength of the radome were verified by the analysis and tests. Beam pattern test, beam control test and beam steering test are performed in an anechoic chamber for the transmitting CSAAS. The results of structural and electromagnetic tests showed that the design objects met the goal successfully.
\end{abstract}

\section{INTRODUCTION}

SATCOM antenna installed on the global hawk, which is the reflector antenna, causes a huge front radome to protect SATCOM antenna. Whereas SATCOM antenna installed on B-2 stealth bomber is a conformal array antenna. Essential planar antenna to compose a stealth aircraft gives a little volume to the aircraft which results in aerodynamic drag reduction, operational efficiency increase and easy structural design, etc. But we barely find the applied aircraft planar SATCOM antenna because the level of difficulties of array antenna design is very high. SATCOM antenna uses geostationary satellite and the distance from geostationary satellite to Korea is $37,000 \mathrm{~km}$ which is very long distance to make it consider the area of the planar antenna important.

This study designed and manufactured transmitting conformal SATCOM array antenna structures to verify ground to satellite communication. The structure and the antennas are integrated into one system and the antenna performance test, beam steering test was done in 
an anechoic chamber.

The location of the antenna was selected on $\mathrm{x}-47$ based aircraft and the aerodynamic loads were induced. Static structural tests were performed to verify the structural strength of the CSAAS and impact load were applied to verify the impact strength.

\section{DEVELOPMENT OF CSAAS}

\subsection{Requirements}

The design objectives of CSAAS are summarized in table 1.

Table 1. Design Object

\begin{tabular}{|c|c|l|l|}
\hline No. & \multicolumn{1}{|c|}{ Item } & \multicolumn{1}{c|}{ Design Objective } \\
\hline 1 & Structural Strength & Carry aircraft skin load \\
\hline 2 & Stiffened Structure & Design stiffened grid \\
\hline 3 & Antenna Frequency & Ku-band \\
\hline 4 & Antenna Type & Tile Type \\
\hline 5 & Antenna Performance & 45 dBW(EIRP) \\
\hline \\
2.2 Structures
\end{tabular}

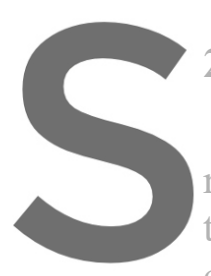

\subsubsection{Design}

The structure is made

radome was selected as
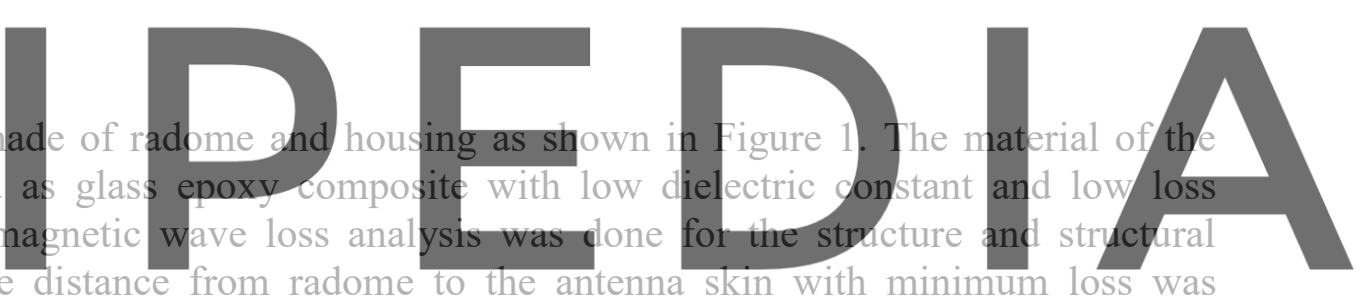

cross section and the distance from radome to the antenna skin with minimum loss was

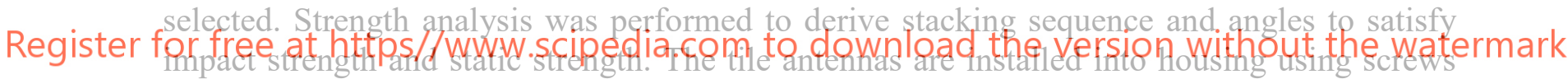
and the connectors are attached to the backside of the housing to support data and power. The angie of side structure is decided considering physical interference when the electronic beam steers. Ventilation air holes are provisioned on the side of the housing for cooling the activating the tile antennas.

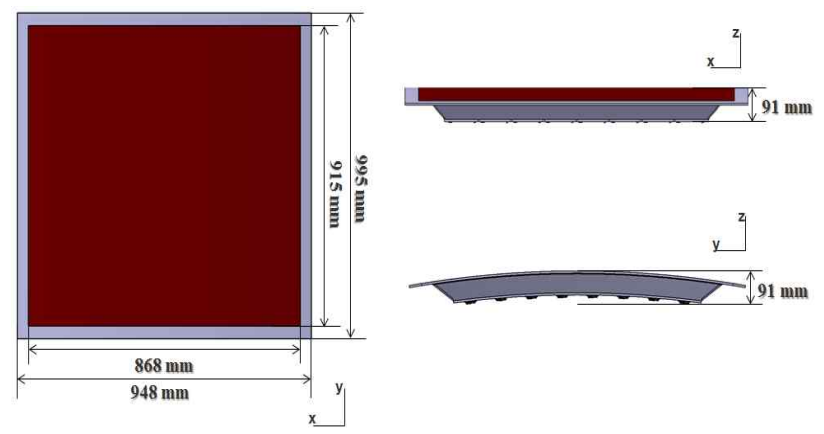

Figure 1. Conformal Array Antenna Structure 


\subsubsection{Fabrication}

Array antenna structures are fabricated using autoclave curing process that includes grids and substructure together. The grids are cut following the grid shape before the curing process and stacked after substructure stacking.

\subsubsection{Verification}

Structural strength tests are performed for the array antenna structures. Out-of-plane directional deflection tests are performed to derive maximum allowable deflection of Tension/Compression/Shear loads. Figure 2 shows shear load strength test for the array antenna structures. Radome and substructure are integrated and fixed to beam structures. One actuator is connected from the bottom for a shear load and strain gages and displacement gages attached on the radome surface. Structural behaviors of the test are compared with simulation results
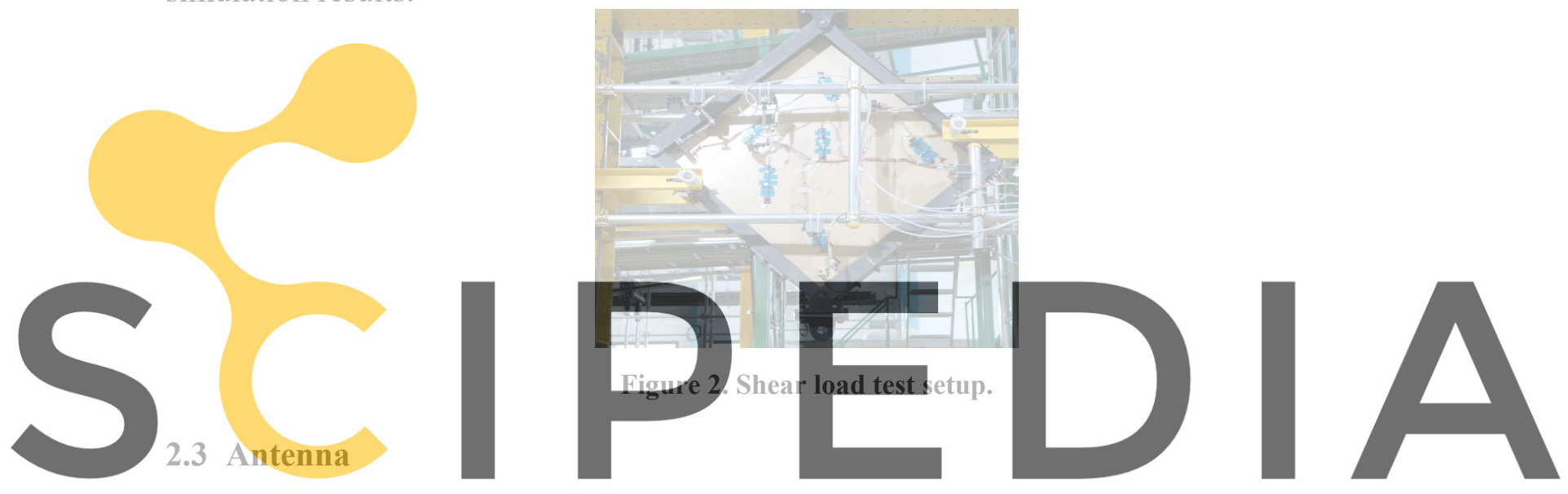

Register for free at https///www.scipedia.com to download the version without the watermark Transmitting tile antenna has one PCB(Printed Circuit Board) and four MLBs(Multi-Layer

Board) stacked under antenna board. The PCB has 22 layered Rogers substrates and core-

chips, power dividers, band path filters and MCU(Main Control Units)s are distributed on it. Transmitting tiles has vertical and horizontal polarizations. Antenna board has core-chips for dual polarization as shown in Figure 3 and LNA(Low Noise Amplifier)s, PS(Phase Shifter)s, and DA(Driving Amplifier)s are located in each core-chips. The tile has 128 core-chips and 8 MCUs that has 1:16 divider.

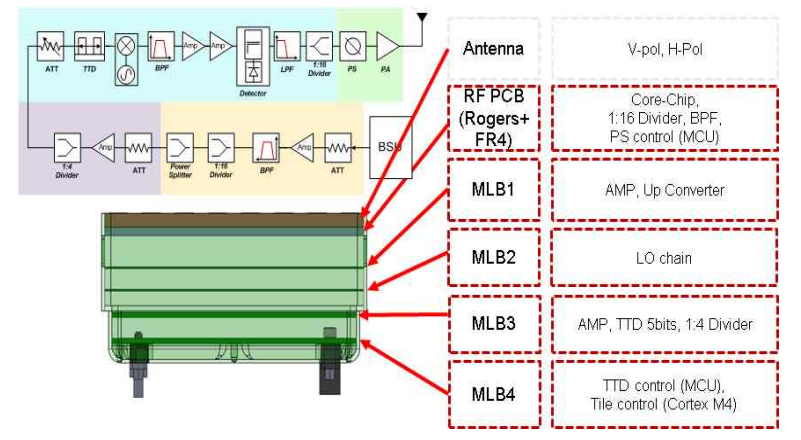

Figure 3. Multi-layered Structure of Transmitting Tile 


\subsubsection{Array Antenna}

Transmitting satellite communication antenna is using Ku-band frequency. The band width is $500 \mathrm{MHz}$. Figure 4 shows transmitting array antenna system consisted of antenna element, cell, tiles and sectors. Four cell of one tile antenna has L-band frequency transferred from $\mathrm{Ku}-$ band. After going through TTD(True Time Delay) and 4:1 power divider the signal goes to sector.

Transmitting signal input starts from analog interface and BSU-T divides after going through ADC(Analog to Digital Converter) as digital signal. DDC/DUC and weighting determines sector level path compensation and polarization direction of transmitting signal. IF analog signals are sent out to sectors.
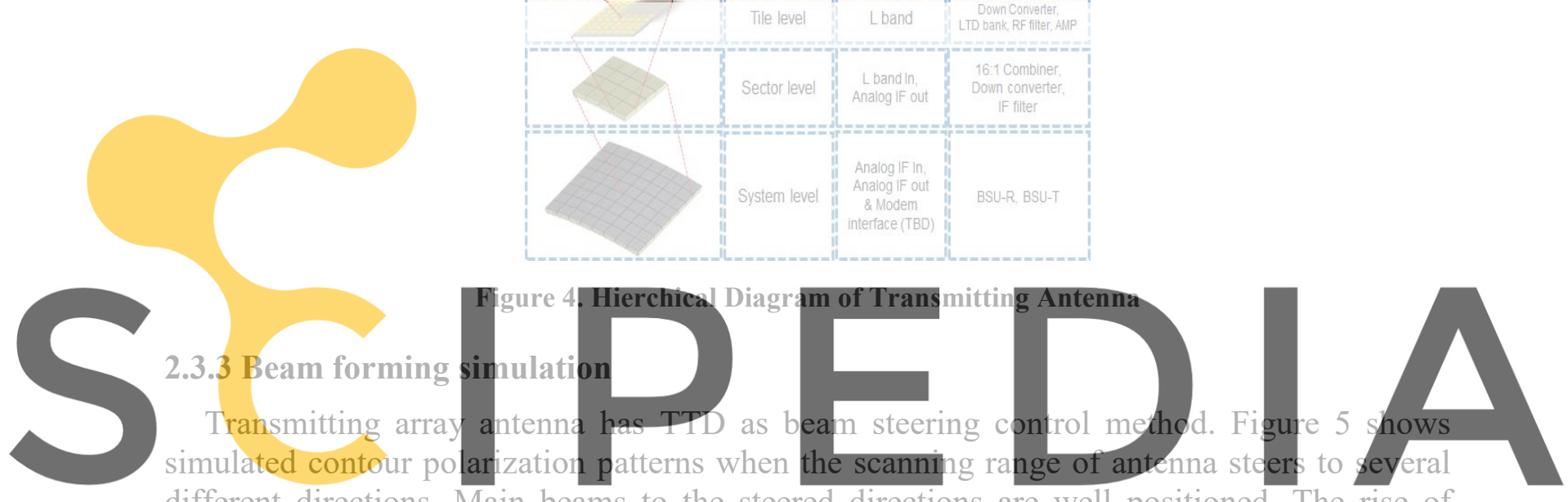

different directions. Main beams to the steered directions are well positioned. The rise of Register fordefobe level appears to the elevation and azimuth angle direction and make vell hiv strand
m Figure 5.

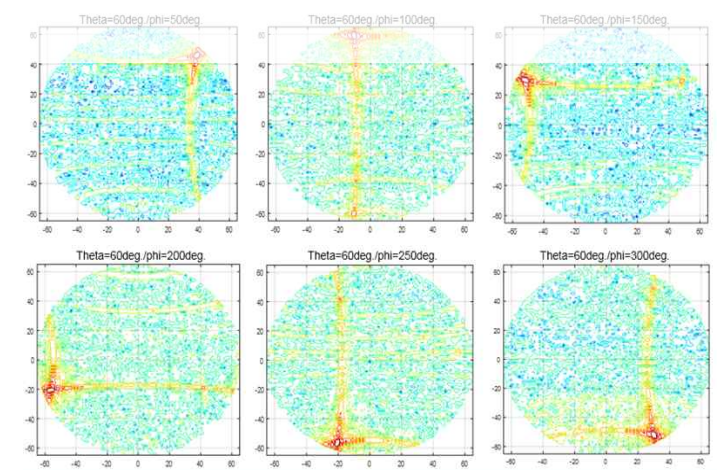

Figure 5. Polarization patterns according to steered main beam direction

\subsubsection{Beam Forming Network}

The use of simple PS(Phase Shifter) to steer beam direction makes beam difference for the frequencies as shown in Figure 6. That's because phase difference between tile antennas depend upon physical distance between tile antennas. Actually the phase difference for the 
physical distance varies according to the operating frequencies. To compensate the time delay of phase path between antennas, TTD(True time delay) device should be used.
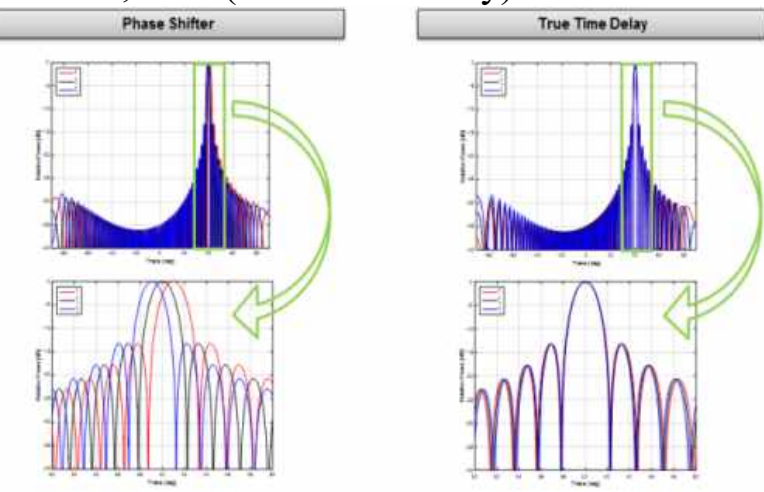

Figure 6. Beam squint comnarision for PS and TTD devices

\subsection{CSAAS Integration}

\subsubsection{Mechanical Integration}

Transmitting tile antennas are installed into the bottom of housings. 64 transmitting tiles are fixed using screws and connectors are hooked to the sectors and BSU. Signal cables and power cable are connected from tile to sector and BSU.
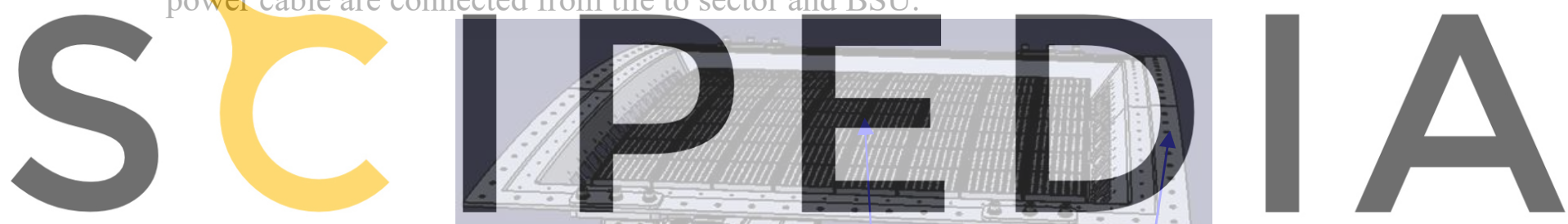

Register for free at https//www.scipedia.com to download the version without the watermark

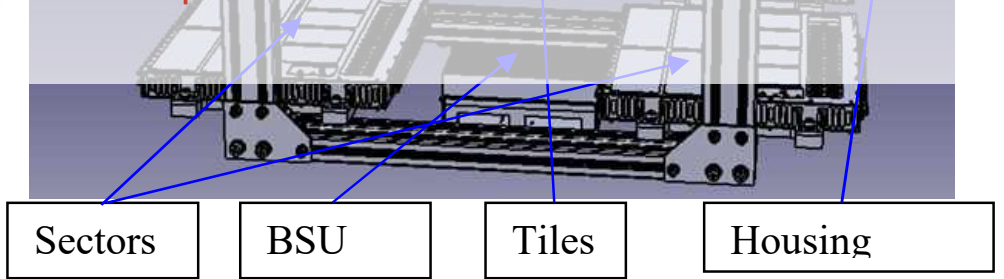

\subsubsection{Antenna System Calibration}

After calibration of each 64 tile antenna, one calibration for one phase of 64 tile antenna system is performed. Phase control devices are applied for the tile level because the calibration of the core-chip in the tiles are finished.

\subsubsection{Antenna System Performance}

Figure 9 shows RF block diagram from tile to element antenna. RF signal from sector goes to 64 tiles and each tile has 1:4 power divider before cell. One cell has polarization signal 1:16 power distributor after TTD, amp, frequency converter and filter and signal goes to antenna element through core-chip. Transmitting array antenna has 4,096 antenna elements, 
8,192 core-chips and 512 frequency converter. The test result for EIRP in an anechoic chamber shows $68 \mathrm{dBW}$. Figure 10 shows far-field horizontal polarization pattern for Azimuth angle $0^{\circ}$ and elevation angle $60^{\circ}$. Figure 11 shows far-field vertical polarization pattern for Azimuth angle $0^{\circ}$ and elevation angle $30^{\circ}$.

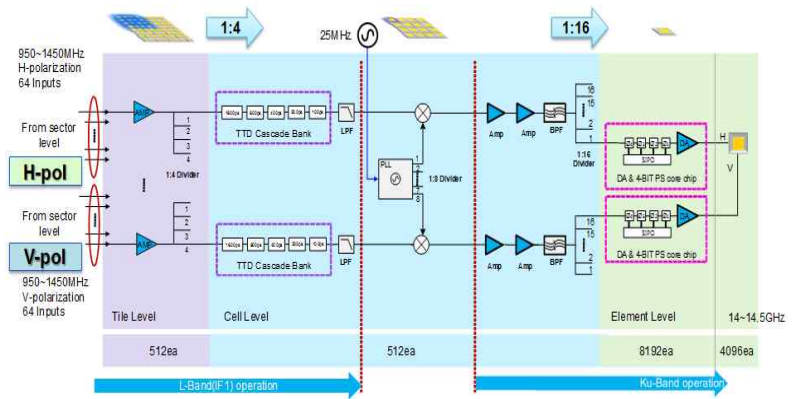

Figure 9. RF Block diagram from Tile Input to Patch Antenna
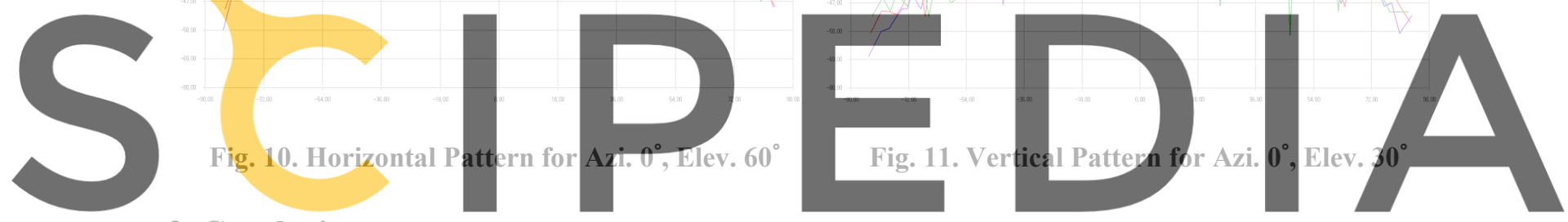

\section{Conclusion}

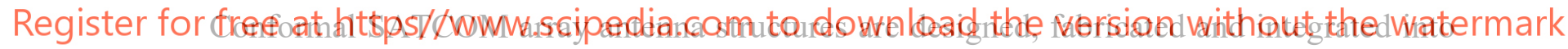

a transmitting conformal array antenna structures that can be installed into an aircraft.

Mechanical performances are satisfied by designing housing as orthogrid structure and mechanical/electromagnetic performance are satisfied by installing tile antennas between grids. Impact strength is satisfied by designing radome as a-sandwich structures. Transmitting antenna can actively steer the beam from $0^{\circ}$ to $60^{\circ}$ maintaining beam-width. We verified all the requirements of CSAAS are met by tests.

\section{REFERENCES}

[1] Baek, S.M., Go, M.G., Kim, M.S. and Joo, Y.S. Structural Design of conformal loadbearing array antenna structures(CLAAS). Advanced Composite Materials, Vol. 25, (2017):29-42.

[2] Mackenzie, Anne I., Electromagnetic Modeling for the Conformal Lightweight Antenna System for Aeronautical Communications Technologies(CLAS-ACT) Program: Final Report. NASA/TM-2019-220293, NASA Langley,(2019). 